\title{
Telescope Array Radar (TARA): First Measurement of EAS Radar Cross-section Upper Limit
}

\author{
Isaac Myers*: \\ Department of Physics and Astronomy, University of Utah \\ E-mail: isaacecosmic.utah.edu \\ John Belz \\ Department of Physics and Astronomy, University of Utah \\ E-mail: belzaphysics.utah.edu
}

TARA (Telescope Array Radar) is a cosmic ray radar detection experiment co-located with Telescope Array (TA), the conventional surface scintillation detector (SD) and fluorescence telescope detector (FD) near Delta, UT. The TARA detector combines a $40 \mathrm{~kW}$ transmitter and high gain transmitting antenna which broadcasts the radar carrier over the SD array and in the FD field of view to a $250 \mathrm{MS} / \mathrm{s}$ DAQ receiver. Data collection began in August, 2013. We have created a novel signal search technique in which the expected (simulated) radar echo of a particular air shower is used as a matched filter template and compared to radio waveforms obtained by triggering the radar DAQ by the fluorescence detector. This technique is used to calculate radar cross-section (RCS) upper limits on triggers that correspond to TA FD events.

The 34th International Cosmic Ray Conference

30 July- 6 August, 2015

The Hague, The Netherlands

\footnotetext{
* Speaker.

$\dagger$ On behalf of the Telescope Array Radar (TARA) and Telescope Array (TA) collaborations.

${ }^{\ddagger}$ For full author list and acknowledgements see http://www.telescopearray.org/images/papers/ICRC2015authorlist.pdf
} 


\section{Introduction}

Investigation of alternate cosmic ray (CR) detection methods is driven by the desire for better statistics. The CR energy spectrum dictates that UHECR event rates are low. Any new technique that measures energy, composition and geometry in a large fiducial volume at lesser cost will likely be successful. Radar detection of cosmic rays [1] is the only active technique being pursued that has the possibility of providing better statistics at the highest energies. Radar has $100 \%$ duty cycle and is a remote-sensing technique that does not require instrumentation of large areas.

TARA improves on other radar experiments in several ways. It is the first CR radar experiment with a dedicated high-power ( $25 \mathrm{~kW}$, typically), continuous wave $54.1 \mathrm{MHz}$ transmitter and high gain TX antenna array [2]. The transmitter (TX) and receiver (RX) are located to the northeast and southwest of the TA SD array, a geometry which directs the TX main lobe over the SD array to maximize coincident event detection (see Figure 1). Co-location with TA is the primary advantage of TARA. Other experiments have observed possible CR radar reflections, but lacked the ability to confirm events with a conventional detector.

\section{TARA Detector}

The TARA transmitter and receiver apparatus have been described in detail in [2]. Briefly, the DAQ and RX antennas are located on the site of the Long Ridge (LR) fluorescence detector (FD). The DAQ sample rate is $250 \mathrm{MS} / \mathrm{s}$ with a passband restricted to the range [40, 80] $\mathrm{MHz}$ by passive hardware filters to remove anthropogenic noise sources. There is also an RF limiter, lightning protector and either a 30 or $40 \mathrm{~dB}$ broadband amplifier on each channel. Two dual-polarized logperiodic dipole antennas (LPDA) feed four DAQ input channels. Each antenna has a horizontal and vertical polarization output.

There are three DAQ trigger modes: FD-trigger, self, and snapshot. FD-triggered data are forced triggers from a low level FD hardware source. The majority of FD triggers are background noise from night sky fluctuations. FD trigger mode only activates when the FD is in operation, and therefore has approximately $10 \%$ duty cycle. The self-trigger is an intelligent mode that uses a bank of five matched filters (MF) and the snapshot trigger is a once-per-minute record used for estimating background noise. All trigger types record 32744 samples ( $\sim 131 \mu$ s duration) in each of four channels. Only FD-trigger and snapshot data analysis and results from a single antenna's horizontal polarization are presented here. The TX antenna was horizontally polarized at the time these data were recorded, therefore we expect that the strongest signal will be received with the same polarization in the receiving antenna. FD-triggered data are our most promising set of triggers because we know that a subset correspond to actual CR events.

\section{Radar Echo Simulation}

A simulation has been developed to calculate the expected radar echo from CR extensive air showers (EAS) [3, 2, 4]. The kernel of the simulation is the bistatic radar equation,

$$
P_{R}=\frac{P_{T} G_{T}}{4 \pi R_{T}^{2}} \sigma \frac{G_{R}}{4 \pi R_{R}^{2}} \frac{\lambda^{2}}{4 \pi}
$$


$P_{R}$ is received power, $P_{T}$ is transmitter power, $G_{R}$ and $G_{T}$ are the receiver and transmitter antenna gains, respectively, $\lambda$ is the radar wavelength, $R_{T}$ is distance between transmitter and target, $R_{R}$ is distance between target and receiver, and $\sigma$ is the radar cross section (RCS). Key parameters such as duration, frequency shift [5], and received power are desired simulation results. Much care has been taken to include detector, atmospheric and CR phenomenological effects.

Full three dimensional TX and RX radiation patterns are simulated and coded as lookup tables [2]. Gaisser-Hillas, NKG, and Nerling's alpha combine to give the free electron density as a function of shower age. The 1976 Standard Atmosphere gives air temperature and density as functions of altitude. The electron recombination/attachment lifetime is also parameterized.

Shower ionization electrons are densest very near the shower core. We approximate the EAS as a short-lived, perfectly conducting thin wire using an approximation to the thin-wire RCS [6] $\sigma_{\mathrm{TW}}$. This model overestimates received power because it neglects the damping effect of free electron collisions with atmospheric molecules [7, 8]. Simulation frequency vs. time is model-independent, being a function of EAS geometry only. The simulation shows that CR echoes are short in duration ( $\sim 10 \mu \mathrm{s}$ ), have large frequency down-shifts on order of the radar frequency, and have the highest power near the end of the signal which is typically near the radar frequency (see Figure 2).

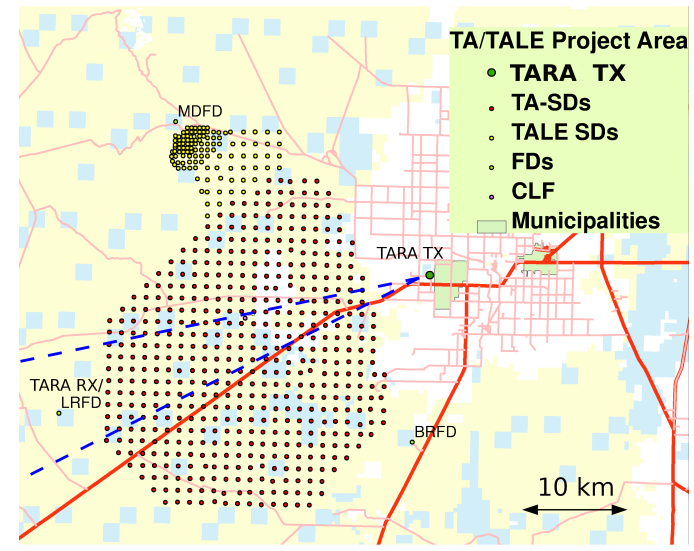

Figure 1: Map of TARA Observatory sites (transmitter and receiver) along with the Telescope Array (TA) detector facilities. Blue lines indicate the transmitter beamwidth $3 \mathrm{~dB}$ below the peak gain.

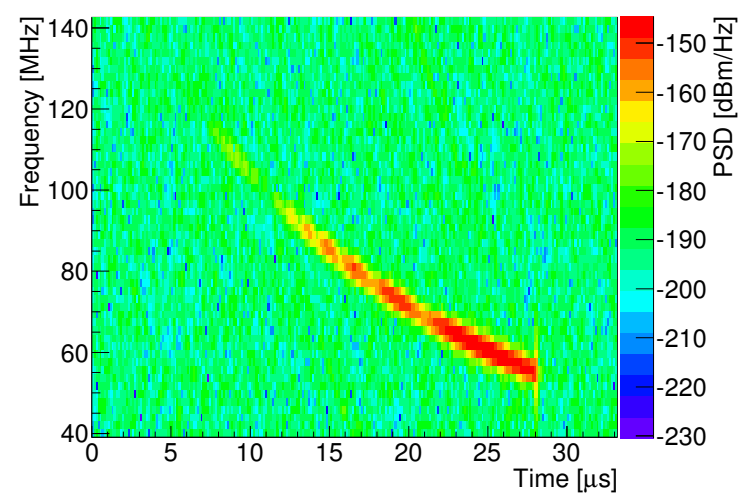

Figure 2: Radar echo spectrogram from a $10^{19} \mathrm{eV}$ shower located midway between transmitter and receiver inclined $30^{\circ}$ out of the plane connecting the two.

\section{Analysis of Reconstructible Triggers}

The first step in FD-triggered data analysis is time matching with reconstructed TA FD events recorded between August, 2013 and ending in April, 2014, with an absolute time difference no greater than $200 \mu \mathrm{s}$. This step decreases the amount of triggers by roughly a factor of 1000 by removing triggers which correspond to background noise or events removed by quality cuts (Table 1). After matching, 1232 events remain with a mean time difference of $33 \mu \mathrm{s}$, where the TARA DAQ lags the reconstructed FD event time. 
Table 1: Long Ridge FD reconstructed events quality cuts. Pseudo-distance is the distance which corresponds to an angular speed (deg./ $\mu \mathrm{s}$ ) of the shower track across the field of view of the detector. The tangent fit $\chi^{2} /$ DOF represents the quality of the fit to the shower track time vs. angle plot, which is used to determine $\Psi$, the angle of the shower in the SDP. (NPE = number of photo-electrons; SDP = shower detector plane; FD geometry has been described in the literature [9]).

\begin{tabular}{|c|c|l|}
\hline Cut Name & Criteria & Description \\
\hline \hline tube fraction & $\geq 3.5 \%$ & data quality, noise reduction \\
\hline good tubes & $\geq 6$ & data quality, noise reduction \\
\hline NPE/degree & $\geq 25$ deg. $^{-1}$ & data quality, sufficient signal \\
\hline pseudo-distance & $\geq 1.5 \mathrm{~km}$ & geometry, shower resolution \\
\hline SDP angle & $<80^{\circ}$ & geometry \\
\hline$R_{p}$ & $R_{p} \geq 1 \mathrm{~km}$ & geometry \\
\hline$\Psi$ & $0^{\circ} \leq \Psi<150^{\circ}$ & geometry \\
\hline$\delta \Psi$ & $<36^{\circ}$ & fit reconstruction, $\Psi$ error \\
\hline tangent fit & $\chi^{2} / \mathrm{DOF}<10$ & fit reconstruction \\
\hline zenith angle & $<70^{\circ}$ & geometry \\
\hline$t_{0}$ & $t_{0}<25.6 \mu \mathrm{s}$ & event occurs in trigger window \\
\hline$R_{p}$ and $t_{0}$ & $!\left(R_{p}<5 \mathrm{~km} \& \& t_{0}<3 \mu \mathrm{s}\right)$ & geometry \\
\hline first tube depth & $150<X_{1}<1200\left[\mathrm{~g} / \mathrm{cm}^{2}\right]$ & geometry, first tube illuminated from reason- \\
& & able depth \\
\hline slant depth & $>150 \mathrm{~g} / \mathrm{cm}^{2}$ & geometry, minimum track length \\
\hline$X_{\max }$ & $400<X_{\max }<1200\left[\mathrm{~g} / \mathrm{cm}^{2}\right]$ & fit reconstruction \\
\hline
\end{tabular}

Before further analysis, events are software filtered with a $54.1 \mathrm{MHz}$ notch filter to remove the carrier which dominates all other frequencies and a $30 \mathrm{MHz}$ high pass filter to remove citizen band (CB) and other spurious noise below $30 \mathrm{MHz}$. Intermittent carriers from anthropogenic sources and short, broadband impulsive noise from unknown sources are present in the passband.

Noise features are accounted for in the analysis chain by estimating backgrounds using snapshots that occur during active FD-trigger observation periods. Distributions of waveform voltage RMS for filtered FD-triggered matched events and filtered snapshots recorded during the August, 2013 FD observation period are in good agreement. An initial eye scan of the 1232 matched events' time- and frequency-domain plots did not result in the observation of waveform or spectral features that differ from snapshots in any statistically significant way. Therefore, if radar echo signal is present in the FD-triggered waveforms, we assume it is below the noise level and expect agreement in the waveform VRMS (voltage RMS) distributions.

Matched filtering (MF) is a digital signal processing technique where a template or ideal waveform is searched in a test waveform. Application of the MF is the calculation of the vector product of the template with the test waveform; the result is a single value, with larger values indicating greater probability that the template is present in the test waveform. To maximize sensitivity the MF is applied beginning at every sample in the waveform trigger time, which is between 48 and 
$101 \mu \mathrm{s}$ in the TARA waveform. The actual DAQ trigger time is $96 \mu \mathrm{s}$, but has been adjusted based on FD and TARA DAQ trigger timing. For each waveform, only the absolute maximum MF value is saved in the course of analysis.

The background MF threshold is calculated for each of the 1292 matched events using as a template the simulated radar echo from the reconstructed event primary energy, core location, geometry, and depth of $X_{\max }$. 400 filtered snapshots are MF analyzed in the defined trigger time range to obtain 400 peak MF responses. The $3 \times$ RMS level from the resultant distribution is the positive detection threshold $\gamma_{3 \mathrm{RMS}}$. There are six snapshots sets that correspond to FD data acquisition periods. Thresholds are calculated for each of the sets and compared to the appropriate matched event.

FD-triggered waveforms' peak MF responses are calculated and compared to their individual thresholds. Those with peak MF responses $\beta_{\text {textevent }}>\gamma_{3 \mathrm{RMS}}$ are considered tentative positive detections. 17 events of 1292 triggers were tagged as positive detections. 17.8 events were expected from the snapshot MF distributions created to calculate $\gamma_{3 \mathrm{RMS}}$. This gives a chance probability of $60 \%$. We conclude that events tagged as positive detections are those with common noise in the passband.

RCS upper limits are calculated by superimposing the simulated echo waveform scaled by $\Lambda$ on snapshots such that $90 \%$ of the snapshots have a peak MF response $\beta$ that exceeds the threshold. The procedure for each of the events which have $\beta_{\text {textevent }}<\gamma_{3 \mathrm{RMS}}$ is to superimpose the eventspecific simulated waveform scaled by $\Lambda$ on 400 snapshots, apply notch and high pass filters, calculate $\beta_{j}$ in the trigger time range for each of the 400 waveforms, choose a new $\Lambda$ and repeat until $90 \%$ of $\beta_{j}$ exceed $\gamma_{3 \text { RMS }}$.

Voltage squared is proportional to power, and power is proportional to the RCS in the bi-static radar equation 3.1, so the number that scales the RCS is $\Lambda^{2}=\Gamma$. The EAS RCS upper limit at the $90 \%$ confidence level is $\sigma_{\mathrm{EAS}}<\Gamma_{90} \cdot \sigma_{\mathrm{TW}}$, where $\Gamma_{90}$ is the scale factor at the $90 \%$ confidence level. Figure 3 shows a core location plot of events with $\Gamma_{90}<0.1$, events to which TARA is most sensitive. The five lowest $\Gamma_{90}$ events have green arrows which begin at the core location and point in the CR direction of origin and have lengths proportional to the zenith angle.

Table 2 summarizes the five lowest $\Gamma_{90}$ events, and the corresponding increase in $\Gamma_{90}$ from systematic error in reconstruction parameters. One observes that the five lowest $\Gamma_{90}$ events to which TARA is most sensitive are highly inclined to minimize polarization angle difference between TX and shower axis and pass through the main lobe of the transmitter.

The last entry in the table, an $11 \mathrm{EeV}$ event with $\Gamma_{90}=0.00077$, is our lowest limit. We calculate the maximum RCS for this event by multiplying the maximum of the simulated shower RCS, using the thin-wire model, by $\Gamma_{90}$. At its peak, this event is equivalent to a $42 \mathrm{~cm}^{2}$ reflective object moving near the speed of light.

\section{Analysis of Nonreconstructible Triggers}

The previous analysis rejects all FD triggers that correspond to nonreconstructible events. This restricts the number of events by requiring a minimum $R_{p}$ of $1 \mathrm{~km}$ (Table 1 ). Events that occur close to the FD pass through the telescope field of view very quickly, so shower evolution and profile are not clearly observed. However, the bistatic radar equation (Equation 3.1) predicts maximum 


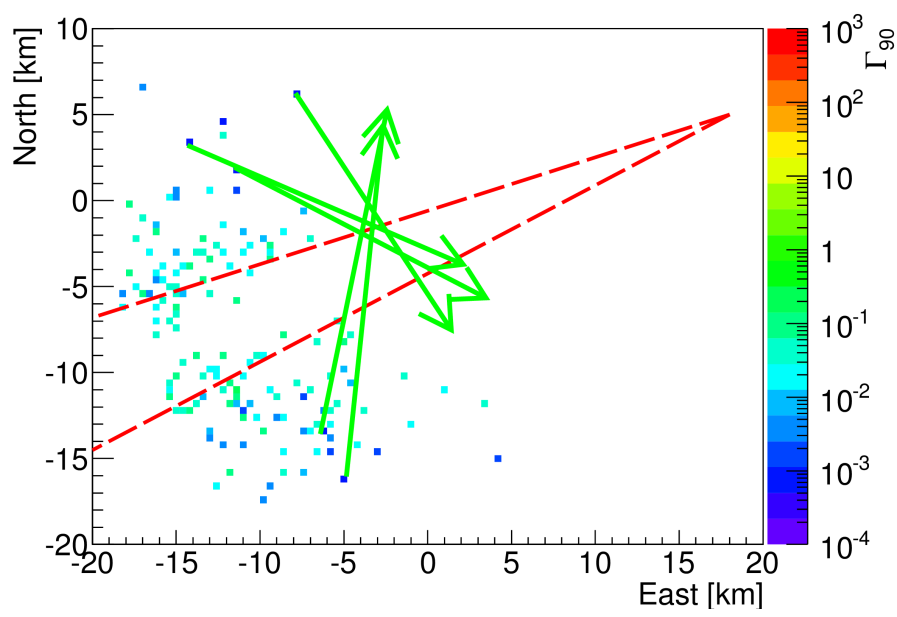

Figure 3: $\Gamma_{90}$ (color scale) for events with $\Gamma_{90}<0.1$. Green arrows begin at the core location of the five lowest $\Gamma_{90}$ events and point in the direction of each event's azimuth. Arrow length is proportional to zenith angle. Red, dashed lines are transmitter antenna beamwidth at the points $3 \mathrm{~dB}$ below the peak.

received power when the target is close to either the transmitter or receiver. There are no events that occur near the transmitter station because it beyond the detection range of the FD, but many events occur close to the FD where the TARA receiver is located.

We wish to conduct a search for evidence of CR radar echoes in FD triggers that fail the $R_{p}$ cut. After time-matching TARA FD triggers with events that fail the $R_{p}$ cut in the same manner as above, 2124 events remain. By definition, these events are not reconstructible, so we rely on the simulated radar echo of Monte Carlo events to create a set of matched filters which will be used in the search. A large set of FD-triggering Monte Carlo events was narrowed down to a total of 673 events by requiring that $E_{0}>3 \times 10^{17} \mathrm{eV}$ and $R_{p}<1 \mathrm{~km}$.

Radar echoes from the Monte Carlo events are simulated and analyzed for chirp slope and duration within the passband. Due to the proximity of the shower and receiver, many of the simulated events are undetectable because they have such large frequency shifts that the radar echoes never descend below $80 \mathrm{MHz}$ into the passband. Duration and slope distributions of the simulated events are shown in Figures 4 and 5. Entries with zero duration or slope are events with echoes above the

\begin{tabular}{|l|c|c|c|c|c|c|}
\hline Date & $\Gamma_{90} \times 10^{4}$ & Energy & Core Loc. & Zen. & Azi. & $X_{\max }$ \\
\hline \hline 20130809 & $8.4+5.0$ & 1.22 & $(-11.5,1.9)$ & 65.7 & 301.6 & 772 \\
\hline 20130816 & $8.8+2.0$ & 1.43 & $(-7.9,6.2)$ & 68.6 & 280.5 & 755 \\
\hline 20130926 & $9.7+2.8$ & 1.38 & $(-14.3,3.2)$ & 54.9 & 299.5 & 837 \\
\hline 20131105 & $9.2+3.9$ & 1.83 & $(-4.8,-16.0$ & 59.6 & 121.4 & 805 \\
\hline 20131202 & $5.2+2.5$ & 11.04 & $(-6.4,-13.6)$ & 62.7 & 114.6 & 859 \\
\hline
\end{tabular}

Table 2: Summary of five lowest $\Gamma_{90}$ events and the increase in $\Gamma_{90}$ due to systematic uncertainty, both multiplied by a factor of $10^{4}$. Reconstructed event parameters are given in the following units: energy [EeV], core location in $(\mathrm{x}, \mathrm{y})$ pairs $[\mathrm{km}]$ relative to TA's central laser facility, zenith and azimuth angles [deg.], and $X_{\max }\left[\mathrm{g} / \mathrm{cm}^{2}\right]$. 
passband.

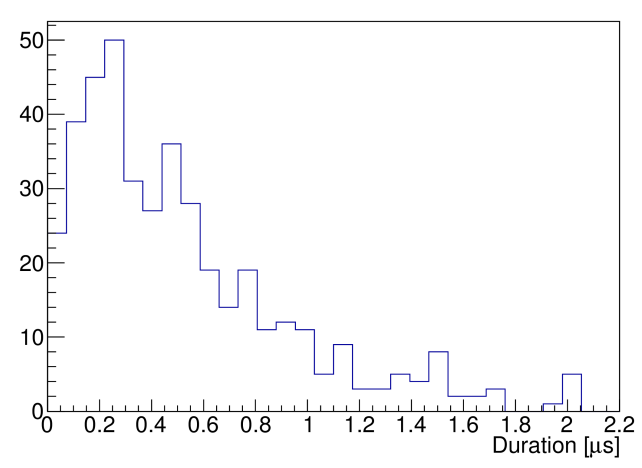

Figure 4: Distribution of radar echo durations within the TARA $[40,80] \mathrm{MHz}$ passband. Duration is defined as the period during which the peak power occurs, with endpoints $10 \mathrm{~dB}$ below the peak.

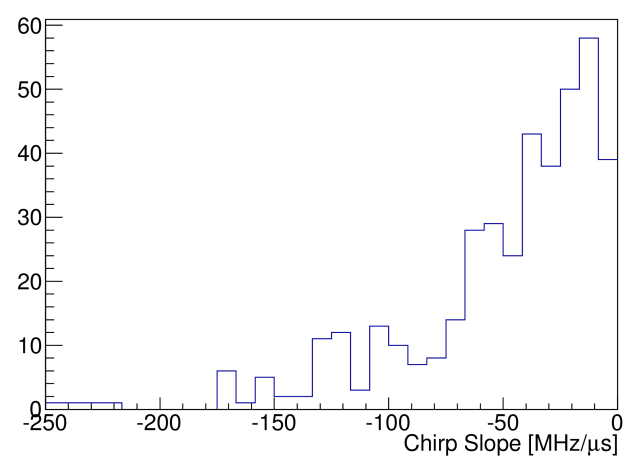

Figure 5: Distribution of radar echo chirp slopes within the TARA $[40,80] \mathrm{MHz}$ passband. The slope is calculated from a simple straight line fit to the power spectrogram.

A set of 21 linear chirp, constant amplitude matched filters (MF) were created to broadly represent the simulated radar echo distributions: MFs are $400 \mathrm{~ns}$ in duration, span $140 \mathrm{MHz}$ of chirp rate in seven discrete steps $(10,20,30,40,50,100$, and $150 \mathrm{MHz} / \mu s)$, and represent three different frequency bands, starting at 80,70 , and $60 \mathrm{MHz}$. A fixed $400 \mathrm{~ns}$ duration will not reduce the MF response in the case where the observed waveform has a chirp-like feature shorter than $400 \mathrm{~ns}$. It will give a lower MF response in the case of a feature lasting longer than $400 \mathrm{~ns}$.

Similar to the previous analysis, a threshold is calculated from the distribution of snapshot MF responses. As before, notch and high pass filters are applied before the MF response is calculated. This analysis differs in that there are 21 values each for MF thresholds from snapshots and individual event MF responses $\left(\beta_{\text {event }}[21], \gamma_{3 \mathrm{RMS}}[21]\right)$. For each event, we compare $\beta_{\text {event }}[i]>\gamma_{3 \mathrm{RMS}}[i]$. The results of this analysis will be presented in the $34^{\text {th }}$ International Cosmic Ray Conference.

\section{Conclusion}

TARA is the first experiment to test CR radar echo detection with a dedicated transmitter in parallel with a conventional, state-of-the-art CR detector. Only the FD-triggered data stream has been analyzed to date; no statistically significant observation of coincidences with TA are reported in comparison with the radio frequency background. However, the non-observation of signal has been used in conjunction with reconstructed event information and detailed CR radar echo simulation to make the first measurement of the RCS upper-limit. Our strongest upper limit is for an $11 \mathrm{EeV}$ UHECR event (see Table 2, fifth row) with $\sigma_{\mathrm{EAS}}<0.00077 \cdot \sigma_{\mathrm{TW}}$ at the $90 \%$ confidence level. At the shower peak, the RCS upper limit is equivalent to a mere $42 \mathrm{~cm}^{2}$ reflective object moving near the speed of light.

\section{References}

[1] P. Blackett et al., Proc. Roy. Soc. A 177, 183 (1941). 
[2] R. Abbasi et al., Nucl. Instrum. Meth. A 767, 322 (2014).

[3] I. Myers, Radar Detection of Ultra High Energy Cosmic Rays, PhD thesis, University of Utah, 2015.

[4] M. A. B. Othman et al., Radar Detection of UHECR Air Showers at the Telescope Array, in Proceedings of the 32nd ICRC, 2011.

[5] D. Underwood, Large Doppler Shift in RADAR Detection of Ultra-high Energy Eosmic Rays, in Radar Conference, RADAR '08. IEEE, pages 1-5, 2008.

[6] J. Crispin and A. Maffett, P. IEEE 53, 833 (1965).

[7] Sir Bernard Lovell, F.R.S., Notes Rec. R. Soc. Lond. 47, 119 (1993).

[8] J. Stasielak et al., ArXiv e-prints (2014).

[9] R. Abbasi et al., Astrophys. J. 622, 910 (2005). 